\title{
Contexto normativo en género $y$ experiencias de mujeres oficiales sobre la vinculación al Ejército Nacional de Colombia ${ }^{1}$
}

https://doi.org/10.21830/9789585380219.05

\author{
Leidy Johana Cabrera-Cabrera ${ }^{2}$ \\ Escuela Militar de Cadetes "General José María Córdova” \\ Ingrid Yuliana Arango Calderón ${ }^{3}$ \\ Escuela de Aviación del Ejército
}

\begin{abstract}
Resumen. En este documento se presentan los principales motivadores que definieron la decisión de 18 mujeres de arma para ingresar al Ejército Nacional, así como su relación con los avances normativos en términos de género en la institución. El estudio tuvo un enfoque mixto y utilizó como técnicas de recolección de información la revisión documental y la entrevista. En general, los motivadores incluyeron su práctica profesional, la vocación de servicio, el ejercicio del liderazgo militar, las expectativas de avance en la carrera militar y el aporte al fortalecimiento institucional y de país. Algunas de las experiencias de las oficiales muestran cierta inseguridad frente a su papel real en las funciones que les son asignadas en relación con su entrenamiento. Si bien ya existe un marco normativo de base que propende a la transversalización del enfoque de género y diferencial en el
\end{abstract}

1 Este capítulo forma parte de los resultados del proyecto de investigación "Diplomado virtual en género y Derechos Humanos para la transformación del Ejército Nacional”, del Grupo de Investigación en Ciencias Militares de la Escuela Militar de Cadetes "General José María Córdova", Bogotá, D. C., Colombia, registrado con el código COL0082556 de Minciencias. Los puntos de vista y los resultados que se presentan en este capítulo pertenecen a los autores y no reflejan necesariamente los de las instituciones participantes.

2 Magíster en Docencia e Investigación Universitaria de la Universidad Sergio Arboleda y especialista en Docencia e Investigación Universitaria de la misma institución. Trabajadora social de la Universidad de La Salle. Directora del Observatorio de Género Militar de la Escuela Militar de Cadetes "General José María Córdova", Bogotá, D. C., Colombia. Orcid: https://orcid.org/0000-0002-6398-6933 Contacto: leidy.cabrera@esmic.edu.co

3 Capitán en el Ejército Nacional de Colombia. Especialista en Ciencias Militares para el Planeamiento del Centro de Educación Militar. Profesional en Ciencias Militares y profesional en Administración Logística de la Escuela Militar de Cadetes "General José María Córdova”. Investigadora de la Escuela Militar de Cadetes "General José María Córdova”, Grupo de Investigación en Ciencias Militares y de la Escuela de Aviación del Ejército, Grupo de Investigación en Aviación Militar. OrciD: https://orcid. org/0000-0002-5995-9262 - Contacto: ingridarangocalderon@cedoc.edu.co 
interior de la Fuerza Pública, requiere de la voluntad de todos los actores determinantes en el Ejército Nacional para su consolidación.

Palabras clave: género; mujer militar; normativa; política.

\section{Introducción}

La transformación del Ejército Nacional de Colombia desde la vinculación de mujeres de arma en el año 2009 ha motivado diferentes estudios que ponen sobre la mesa el asunto de género como una oportunidad de avance hacia la democratización de la profesión militar. En este estudio se describen los principales motivadores que definieron la decisión de 18 mujeres de arma para ingresar al Ejército Nacional, así como los avances normativos en términos de género en la institución. Con este propósito se analiza la incorporación del enfoque de género en las entidades que forman parte del Estado colombiano, la cual ha sido respaldada por un conjunto de referentes normativos, creados en el marco del Sistema Internacional de Derechos Humanos para el reconocimiento y la garantía de los derechos de las mujeres en su diversidad. Estos instrumentos, al ser ratificados por Colombia, han generado una serie de compromisos institucionales, los cuales se deben acoger y materializar en normatividad, políticas públicas, planes, programas y proyectos viables y sostenibles (Ministerio de Defensa, 2018).

Los resultados que se presentan en este capítulo corresponden a un estudio mixto que utilizó como técnicas de recolección de información la revisión documental y la entrevista, en el entendido de que la incorporación de mujeres puede transformarse en una oportunidad para problematizar otras dimensiones de la institución. Para esta investigación es claro que el estudio sobre temas de género en el ámbito militar, específicamente sobre la vinculación de las mujeres de arma, necesita ejes que contemplen la toma de decisiones en términos de la transversalización del enfoque de género en la Fuerza.

En este sentido, se plantearon las siguientes preguntas de investigación: ¿Cuáles fueron los principales motivadores que definieron la decisión de 18 mujeres de arma para ingresar al Ejército Nacional? y ¿̨uál es su relación con el marco normativo internacional, nacional e institucional sobre género? Para 
responderlas, en la primera parte del capítulo se hace un acercamiento teórico que permite comprender la vinculación de las mujeres de arma en perspectiva de género. Posteriormente, se describe la metodología utilizada y se presentan los resultados.

\section{Estereotipos}

Uno de los estereotipos que a lo largo de la historia se ha mantenido con más fuerza y fiabilidad es el del sexo. En múltiples trabajos, algunos ya clásicos y otros más cercanos se encuentran descripciones de los grupos de hombres y de mujeres que engloban características similares en contextos tan diversos como América del Norte y del Sur, Asia, África, Europa y Australia. Estas descripciones retratan a las mujeres como sensibles, cálidas, dependientes y orientadas a la gente, en tanto que a los hombres se les ve dominantes, independientes, orientados hacia el trabajo y agresivos (Williams \& Best, 1982).

Tanto mujeres como hombres tienden a actuar de forma apropiada con sus roles y todos hemos acabado por olvidar los efectos reales del desempeño de estos. Se llega a la conclusión de que las diferencias visibles de sus comportamientos demuestran que los unos están, por naturaleza, orientados hacia el trabajo, mientras que las otras están orientadas hacia las relaciones interpersonales, en vez de comprender que se hace por inercia y tradición estereotipadas (Gavaldón, 1999). Así, los estereotipos de género pueden comprenderse como creencias consensuadas sobre las diferentes características de los hombres y las mujeres en nuestra sociedad. Este conjunto de creencias que atañen a las categorías hombre y mujer, que llamamos género, tiene una gran influencia en el individuo, en su percepción del mundo y de sí mismo y en su conducta (Gavaldón, 1999).

Si el objetivo es crear una sociedad donde el sexo de las personas no determine el papel que van a desempeñar en el terreno profesional, tan importante es que la mujer acceda a roles considerados tradicionalmente masculinos, como que los hombres accedan a roles considerados femeninos (Gavaldón, 1999).

Es claro que la mujer tiene unas características propias que la definen y que coinciden con el perfil que se requiere hoy en día para los líderes del siglo XXI. El liderazgo femenino va íntimamente unido a la idea de que la mujer 
necesita satisfacer una doble necesidad: poder realizarse en el campo familiar y en el laboral, sin tener que renunciar a ninguno de los dos (Pulido, 2014).

Desde hace décadas, hombres y mujeres fueron asignados, respectivamente, a dos esferas sociales definidas claramente: la pública y la privada, teniendo la primera un valor político. Por lo tanto, la esfera donde históricamente se desarrollaban las cualidades asociadas a las mujeres fue, durante mucho tiempo, irrelevante para la opinión pública y el mercado. Lo anterior, que constituye el origen de la concepción del género, ha condicionado el acceso de la mujer al ámbito laboral y más aún a puestos de liderazgo y toma de decisiones (Medina-Vicent, 2015).

Tal como lo aclara Medina-Vicent (2015), no se trata de que las mujeres lideren diferente por el hecho de ser mujeres, sino que los distintos procesos de socialización han conducido a una identidad de género concreta que lleva, en el caso de las mujeres, a constituirse en seres sensibles, emotivos y cooperativos, lo cual se va a ver reflejado en una particular forma de liderar, diferente a la de los hombres, cuya socialización está asociada a características como el control, la agresividad, la independencia, etc.

\section{Vinculación de las mujeres de arma y perspectivas de género}

En el contexto militar en Colombia, las mujeres se pueden desempeñar actualmente como oficiales o suboficiales en todas las ramas de la Fuerza Pública, y en distintas especialidades. En enero de 2009 ingresó a la Escuela Militar de Cadetes "General José María Córdova” la primera promoción mixta, de la cual se graduaron 48 de las 62 cadetes en diciembre de 2011 (Camacho, 2014).

La vinculación de las mujeres al Ejército Nacional de Colombia, como oficiales de arma, permite destacar la importancia de la investigación sobre el lugar que tiene el liderazgo como eje de formación fundamental para la institución castrense. Sin embargo, a pesar de las políticas y prácticas de igualdad de oportunidades y gestión de la diversidad, la presencia de mujeres es fundamentalmente desafiante en un nivel cultural / simbólico (Tepe et al., 2016; Woodward \& Winter, 2006). 


\section{Metodología}

El estudio realizado incluyó un enfoque metodológico mixto. Inicialmente, se realizó un análisis cuantitativo del marco normativo orientado hacia el enfoque de género en los ámbitos internacional, nacional y sectorial, de acuerdo con el documento Politica pública sectorial de transversalización del enfoque de género para el personal uniformado de la Fuerza Pública 2018-2027.

Posteriormente, se realizó una entrevista estructurada a 18 mujeres oficiales de arma, en la cual se aborda de manera específica su experiencia en el proceso de vinculación a la Fuerza. Las 18 mujeres militares entrevistadas tenían edades entre los 23 y 30 años, con un promedio de 26 años. El año de vinculación al Ejército Nacional de estas mujeres osciló entre 2009 y 2015.

\section{Resultados}

Los resultados se enmarcan en dos ejes. Por un lado, se presentan los resultados sobre el marco normativo, que permitió ubicar la discusión sobre género, desde lo establecido internacionalmente hasta lo que se plantea en términos institucionales. Por otro lado, se describen las experiencias y los determinantes que describen 18 mujeres de arma sobre su experiencia y vinculación al Ejército Nacional.

\section{Análisis del marco normativo orientado hacia el enfoque de género}

En la revisión desarrollada se contabilizaron instrumentos normativos que se publicaron entre 1979 y 2018. En la figura 1 se presenta la dinámica temporal de la emisión de instrumentos normativos de forma comparativa entre los ámbitos internacional, nacional y sectorial.

En el ámbito internacional, el interés de los gobiernos en general se despertó antes de 1990. No obstante, de forma permanente se han generado instrumentos normativos que son, por cierto, referentes para los propios de cada país. Siguiendo un patrón parecido, en Colombia los instrumentos normativos también comenzaron a conocerse desde antes de 1990 y tuvieron 


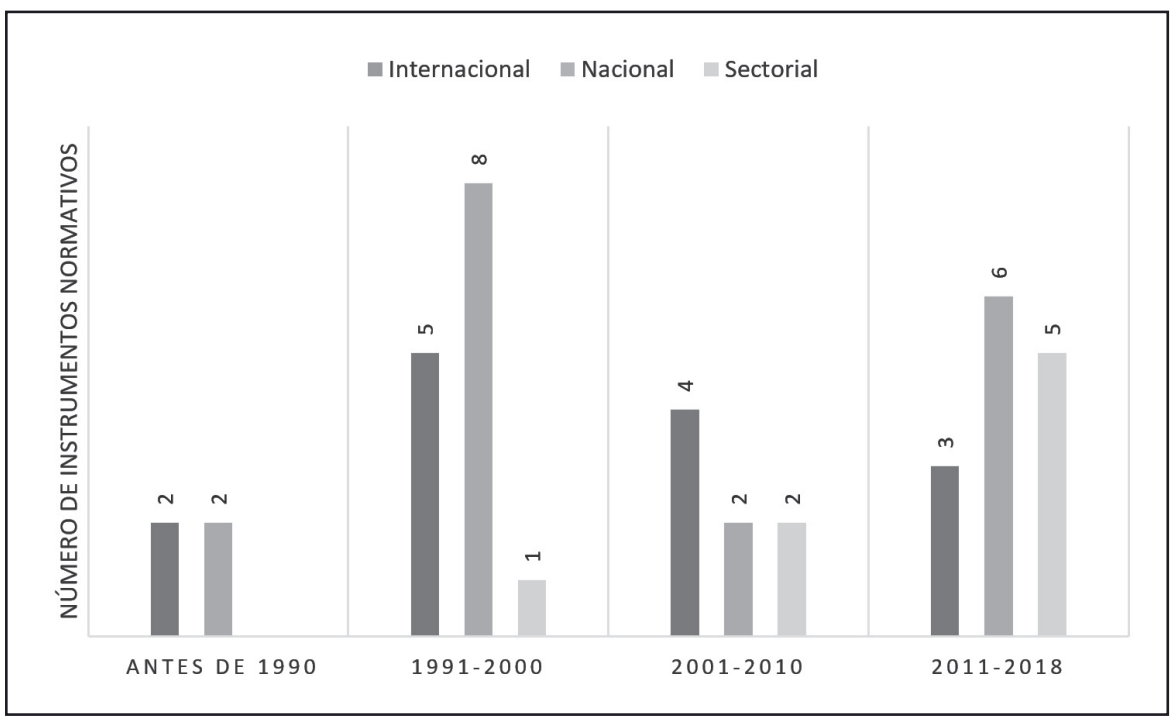

Figura 1. Marco normativo sobre género.

Fuente: Elaboración propia.

un importante incremento entre ese año y el 2000. De forma similar, en los últimos ańos el tema ha requerido de nuevas normas, lo cual explicaría un número relativamente importante de instrumentos normativos en la última década. Por su parte, en el sector defensa estos instrumentos normativos han venido en franco aumento desde hace treinta años, lo cual indica claramente el interés de las Fuerzas Militares en mantener una coherencia clara con la normatividad internacional y nacional con respecto a las políticas de género.

Considerando que la cantidad de instrumentos normativos mencionados es un indicador limitado a una gran categoría de género, es importante desarrollar un análisis más profundo a la luz de categorías que han emergido desde el comienzo de la emisión de instrumentos normativos hace más de treinta ańos. Estas categorías son: discriminación, violencia, derechos e igualdad, enfoque de género y transversalización. Por tal razón, a continuación se listan tales instrumentos normativos y posteriormente se presenta una tabla que permite visibilizar el o los enfoques principales de cada uno de ellos a partir de cada categoría emergente mencionada. 
En total, se encontraron cuarenta instrumentos normativos:

1. Convención sobre la eliminación de todas las formas de discriminación contra la mujer (CEDAw) (1979).

2. Convención contra la tortura y otros tratos o penas crueles, inhumanas y degradantes (1984).

3. Convención interamericana para prevenir, sancionar y erradicar la violencia contra la mujer - Convención de Belem do Para (1994).

4. Conferencia Mundial de la Mujer de las Naciones Unidas en Beijing (1995).

5. Consejo Económico y Social de las Naciones Unidas (1997).

6. Resolución 1325 del Consejo de Seguridad de las Naciones Unidas (2000).

7. Protocolo para prevenir, reprimir y sancionar trata de personas, especialmente de mujeres y niños (2000).

8. Resolución 1820 del Consejo de Seguridad de las Naciones Unidas (2008).

9. Resolución 1888 del Consejo de Seguridad de las Naciones Unidas (2009).

10. Resolución 1889 del Consejo de Seguridad de las Naciones Unidas (2009).

11. Resolución 1960 del Consejo de Seguridad de las Naciones Unidas (2010).

12. Resolución 2106 del Consejo de Seguridad de las Naciones Unidas (2013).

13. Resolución 2122 del Consejo de Seguridad de las Naciones Unidas (2013).

14. Resolución 2242 del Consejo de Seguridad de las Naciones Unidas (2015).

15. Constitución Política de Colombia (1991).

16. Ley 51 de 1981.

17. Ley 82 de 1993.

18. Ley 248 de 1995.

19. Ley 581 de 2000. 
20. Sentencia C-371 de 2000.

21. Ley 679 de 2001.

22. Ley 731 de 2002.

23. Ley 750 de 2002.

24. Ley 800 de 2003.

25. Ley 1146 de 2007.

26. Ley 1257 de 2008.

27. Ley 1448 de 2011.

28. Resolución 459 de 2012.

29. Ley 1719 de 2014 .

30. Ley 1761 del 6 de julio 2015.

31. Decreto N. 1480 del 5 de agosto de 2014.

32. Conpes 161 del 12 de marzo de 2013.

33. Decreto 1790 de 2000.

34. Política integral de Derechos Humanos (DD. Нн.) y Derecho Internacional Humanitario (DiH) del Ministerio de Defensa Nacional (2007).

35. Política de derechos sexuales y reproductivos, equidad y violencia basada en género, salud sexual y reproductiva, con énfasis en $\mathrm{V}_{\mathrm{IH}}$ (2010).

36. Directiva N. ${ }^{\circ} 11$ del 21 de julio de 2010.

37. Protocolo de la Fuerza Pública para la prevención y respuesta a la violencia sexual, particularmente en relación con el conflicto armado y su cartilla operativa (2015).

38. Directiva Permanente N. ${ }^{\circ} 30$ del 10 de marzo de 2015.

39. Directiva Permanente 20151110000277-Mdn-CGFMJEMCSemcfj-Jedhdih-2.

40. Política pública sectorial de transversalización del enfoque de género para el personal uniformado de la Fuerza Pública.

En la tabla 1 se vincula la lista anterior a través del número de cada norma. De igual forma, es importante aclarar que la categoría violencia incluye actos relacionados como explotación y pornografía. Adicionalmente, se incluyó una 
categoría denominada general para indicar actos normativos que vinculan a diferentes grupos e incluyen a la mujer, al igual que otros actos normativos que implican seguimiento a acuerdos previos.

Tabla 1. Categorías principales de los instrumentos normativos hallados

\begin{tabular}{|c|c|c|c|c|c|c|c|c|}
\hline Década & 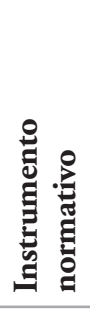 & 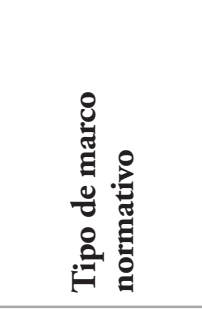 & 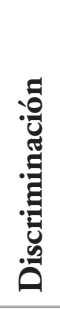 & $\frac{. \frac{\pi}{0}}{\frac{0}{0}}$ & 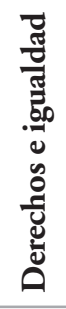 & 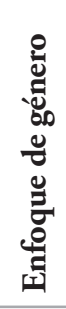 & 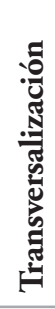 & 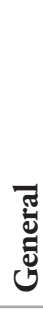 \\
\hline Antes de 1990 & 1 & Internacional & $\mathrm{x}$ & & & & & \\
\hline Antes de 1990 & 2 & Internacional & & $\mathrm{x}$ & & & & \\
\hline $1991-2000$ & 3 & Internacional & & $\mathrm{x}$ & & & & \\
\hline $1991-2000$ & 4 & Internacional & & $\mathrm{x}$ & $\mathrm{x}$ & $\mathrm{x}$ & $\mathrm{x}$ & \\
\hline $1991-2000$ & 5 & Internacional & & & & & $\mathrm{x}$ & \\
\hline $1991-2000$ & 6 & Internacional & & $\mathrm{x}$ & $\mathrm{x}$ & $\mathrm{x}$ & $\mathrm{x}$ & \\
\hline $1991-2000$ & 7 & Internacional & & $\mathrm{x}$ & $\mathrm{x}$ & & & $\mathrm{x}$ \\
\hline $2001-2010$ & 8 & Internacional & & $\mathrm{x}$ & & & & \\
\hline $2001-2010$ & 9 & Internacional & & $\mathrm{x}$ & & & & \\
\hline $2001-2010$ & 10 & Internacional & & & & $\mathrm{x}$ & $\mathrm{x}$ & \\
\hline $2001-2010$ & 11 & Internacional & & $\mathrm{x}$ & & & & \\
\hline $2001-2010$ & 12 & Internacional & & & $\mathrm{x}$ & & & \\
\hline $2001-2010$ & 13 & Internacional & & & & & & $\mathrm{x}$ \\
\hline 2001-2010 & 14 & Internacional & & & & & $\mathrm{x}$ & $\mathrm{x}$ \\
\hline Antes de 1990 & 15 & Nacional & $\mathrm{x}$ & & $\mathrm{x}$ & & & $\mathrm{x}$ \\
\hline Antes de 1990 & 16 & Nacional & $\mathrm{x}$ & & & & & \\
\hline $1991-2000$ & 17 & Nacional & & & $\mathrm{x}$ & & & \\
\hline $1991-2000$ & 18 & Nacional & & $\mathrm{x}$ & & & & \\
\hline
\end{tabular}




\begin{tabular}{|c|c|c|c|c|c|c|c|c|}
\hline Década & 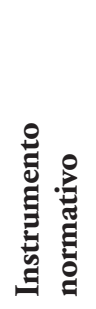 & 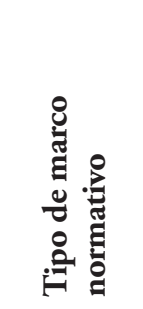 & 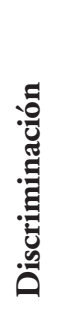 & $\frac{\frac{\pi}{0}}{\frac{0}{0}}$ & 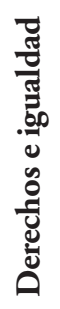 & 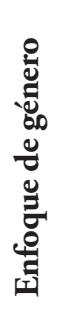 & 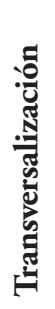 & 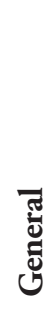 \\
\hline $1991-2000$ & 19 & Nacional & & & $\mathrm{x}$ & & & \\
\hline $1991-2000$ & 20 & Nacional & & & $\mathrm{x}$ & & & \\
\hline $2001-2010$ & 21 & Nacional & & $\mathrm{x}$ & & & & \\
\hline $2001-2010$ & 22 & Nacional & & & $\mathrm{x}$ & & & \\
\hline $2001-2010$ & 23 & Nacional & & & $\mathrm{x}$ & & & \\
\hline $2001-2010$ & 24 & Nacional & & $\mathrm{x}$ & & & & \\
\hline $2001-2010$ & 25 & Nacional & & $\mathrm{x}$ & & & & \\
\hline $2001-2010$ & 26 & Nacional & & $\mathrm{x}$ & & & & \\
\hline 2011-2018 & 27 & Nacional & & $\mathrm{x}$ & $\mathrm{x}$ & & & \\
\hline 2011-2018 & 28 & Nacional & & $\mathrm{x}$ & & & & \\
\hline 2011-2018 & 29 & Nacional & & $\mathrm{x}$ & & & & \\
\hline 2011-2018 & 30 & Nacional & & $\mathrm{x}$ & & & & \\
\hline 2011-2018 & 31 & Nacional & & $\mathrm{x}$ & $\mathrm{x}$ & & & \\
\hline $2011-2018$ & 32 & Nacional & $\mathrm{x}$ & $\mathrm{x}$ & $\mathrm{x}$ & $\mathrm{x}$ & $\mathrm{x}$ & \\
\hline $1991-2000$ & 33 & Sectorial & & & & & & $\mathrm{x}$ \\
\hline $2001-2010$ & 34 & Sectorial & & & & & & $\mathrm{x}$ \\
\hline $2001-2010$ & 35 & Sectorial & & $\mathrm{x}$ & $\mathrm{x}$ & $\mathrm{x}$ & $\mathrm{x}$ & \\
\hline $2001-2010$ & 36 & Sectorial & & $\mathrm{x}$ & & & & \\
\hline 2011-2018 & 37 & Sectorial & & $\mathrm{x}$ & & & & \\
\hline 2011-2018 & 38 & Sectorial & & & $\mathrm{x}$ & $\mathrm{x}$ & & \\
\hline 2011-2018 & 39 & Sectorial & & $\mathrm{x}$ & & $\mathrm{x}$ & & \\
\hline 2011-2018 & 40 & Sectorial & & & & & $\mathrm{x}$ & \\
\hline
\end{tabular}

Fuente: Elaboración propia. 
La tabla 1 permite tener una mayor profundidad de análisis. Es así como claramente se aprecia una tendencia en la normatividad internacional que se repite parcialmente en la nacional. Esta tendencia muestra que las primeras normas en temas de género estuvieron orientadas hacia la discriminación (4 menciones) y poco después hacia la violencia (19 menciones) y el reconocimiento de derechos y de la igualdad (19 menciones). Posteriormente, emergió el concepto de enfoque de género (6 menciones) y finalmente el de transversalización (6 menciones).

De forma interesante, y en contraste con lo observado en la normatividad internacional y nacional, en el sector defensa se han generado instrumentos normativos que vinculan la violencia, los derechos y la igualdad, el enfoque de género y la transversalización de un modo muy cercano en el tiempo. Esto último indica claramente el interés sectorial por abordar el tema de género de una manera integral y en coherencia con el progresivo aumento de personal femenino en las filas.

\section{Experiencias}

Este grupo de resultados ubica la experiencia de 18 mujeres oficiales del Ejército Nacional. Del total de entrevistadas, una pertenece al arma de Artillería, tres al arma de Aviación, tres al arma de Caballería, una al arma de Comunicaciones, una al arma de Infantería, tres al arma de Inteligencia y seis al arma de Logística.

Frente a la pregunta ¿Qué expectativa tenía antes de ingresar al Ejército Nacional?, las oficiales mostraron su interés en aprender sobre la milicia por la inspiración de un familiar, en aprovechar la oportunidad, en un desarrollo profesional que les permitiera liderar, servir y sobresalir incluso como Generales de la República en equidad con los hombres.

Cuando se planteó la pregunta ¿Qué la motivó a vincularse al Ejército Nacional?, las oficiales mencionaron el gusto por la disciplina, la posibilidad de servir a la patria, un referente familiar normalmente masculino, el deporte y la estabilidad laboral.

Con respecto a su Experiencia y trayectoria en el Ejército Nacional, las oficiales indican satisfacción y orgullo ante las funciones desempeñadas, que, 
según señalan, son bastantes y variadas. Por ejemplo, indican la realización de cursos, logros deportivos, mando de tropa, instrucción, docencia, neutralización de criminales, acciones administrativas, aprendizaje de idiomas, capacitación en el exterior, entre otros.

Lo más valorado de la profesión militar por parte de este grupo de mujeres oficiales es el desarrollo profesional y la vocación requerida para ello. Así, ellas resaltan el aprendizaje, la formación integral, el sacrificio, el amor por el país, la posibilidad de servicio, los amigos, las oportunidades brindadas, la estabilidad laboral y la capacidad del Ejército Nacional para reinventarse y reevaluar los escenarios.

Las anécdotas mencionadas por las oficiales son igualmente muy variadas. Todas ellas sugieren una profunda satisfacción y orgullo. Así, se mencionan logros profesionales en la lucha contra la delincuencia, en el desempeño como instructoras, en el agradecimiento y confianza de subalternos y superiores, en el servicio a la comunidad y en el liderazgo.

Ante la pregunta ¿Cómo ve al Ejército Nacional en unos diez años con respecto a la vinculación de mujeres?, las oficiales afirman que lo ven en términos de mejora continua, con mayor número de mujeres, más acoplada al personal femenino, y resaltan que las mujeres continuarán aportando de la misma forma que lo hacen los hombres, con más equidad de género, inclusión y respeto por la mujer.

Finalmente, cuando se indagó sobre el mensaje que les darían a futuras mujeres militares interesadas en vincularse, ellas respondieron con orgullo frente a la institución, la importancia del amor, la pasión, la vocación, la formación integral, el sacrificio, la responsabilidad, el empoderamiento, los valores y la familia.

\section{Discusión}

El contexto normativo sin duda marca una evolución hacia los temas de género que desde el escenario militar se está asumiendo de manera estratégica. Si bien se ha avanzado, es necesario vincular en el quehacer militar lo plasmado en la política sectorial. En los documentos analizados se pone de relieve la 
importancia de abordar el quehacer militar con un enfoque de género, frente a lo cual las mujeres reconocen su vocación militar con orgullo.

Sin embargo, algunas de las experiencias de las oficiales muestran cierta inseguridad frente a su papel real en las funciones que les son asignadas, las cuales en algunos casos no corresponden con las tareas recibidas en su entrenamiento. Tanto en la toma de decisiones como en el campo específico de las funciones que desarrollan estas mujeres existe una cultura de género que requiere de atención directa para que sea comprendida, de tal manera que se desmitifiquen ciertas tareas sobre lo que significa género para el Ejército Nacional y se genere una discusión aplicada sobre lo que ya está establecido en las normativas.

La tarea es clara, ya existe un marco normativo de base que propende a la transversalización del enfoque de género y diferencial en el interior de la Fuerza Pública, a través de la promoción de la equidad de género, la igualdad de oportunidades y la cero tolerancia frente a las violencias basadas en género, con el fin de eliminar las brechas de desigualdad, fortalecer la estructura institucional, la cualificación y la competencia del personal, y cumplir con la misión de la Fuerza Pública en clave de género (Ministerio de Defensa Nacional, 2018). Si bien no es algo sencillo, sí se trata de un proceso que requiere de la voluntad de todos los actores determinantes en el Ejército Nacional para su consolidación.

\section{Conclusiones}

Los lineamientos normativos hacen especial énfasis en la importancia de incluir en el proceso de enseñanza-aprendizaje la cultura de la equidad para fortalecer el ejercicio profesional, lo cual implica reconocer el aporte de las mujeres a la Fuerza.

La experiencia de las mujeres oficiales muestra elementos importantes que ellas han destacado, tales como su práctica profesional, la vocación de servicio, el ejercicio del liderazgo militar y, sobre todo, las expectativas de avance en la carrera militar y el aporte que cada una de ellas hace al fortalecimiento institucional y de país. 


\section{Referencias}

Camacho Zambrano, C. M. (2014). Ejército, feminidades y géneros performativos: Las experiencias de ser mujer y militar en la Escuela Militar de Cadetes "General José María Córdova" [Tesis de Maestría, Pontificia Universidad Javeriana]. Repositorio PUJ. https://repository. javeriana.edu.co/bitstream/handle/10554/14323/CamachoZambranoCarolinaMaria. pdf?sequence $=3$

Gavaldón, B. G. (1999). Los estereotipos como factor de socialización en el género. Comunicar.

Medina-Vicent, M. (2015). Aproximación al estudio del liderazgo femenino a través del modelo transformacional. Universitat Jaume-I. http://repositori.uji.es/xmlui/ bitstream/handle/10234/158386/Aproximacion_al_estudio_del_Liderazgo_ Fe.pdf?sequence $=18$ is Allowed $=y$

Pulido Rivera, S. (2014). Liderazgo y mujer. Dedica. Revista de Educação e Humanidades, (6), 273-283. https://revistaseug.ugr.es/index.php/dedica/article/view/6979/6092

Tepe, V., Yarnell, A., Nindl, B. C., Van Arsdale, S., \& Deuster, P. A. (2016). Women in combat: Summary of findings and a way ahead. Military Medicine, 181(suppl_1), 109-118.

Williams, J., \& Best, D. L. (1990). Measuring sex stereotypes: A multination study. Sage.

Woodward, R., \& Winter, T. (2006). Sexing the soldier: The politics of gender and the contemporary British Army. Routledge. 